\title{
हिमाचल प्रदेश की शैव-परम्परा का ऐतिहासिक एवं सांगीतिक अध्ययन
}

\section{Sarswati ${ }^{1}$ and Prof. Jeet Ram Sharma ${ }^{2}$}

Research Scholar, Department of Music, Himachal Pradesh University, Shimla Professor, Department of Music, Himachal Pradesh University, Shimla

\section{ABSTRACT}

Himachal Pradesh is known for its unique culture and traditions. Shiva is worshiped in all the districts of Himachal. Lord Shiva is worshiped in one form or another. Historians have described Shaivadharma on the baisi of coins used by the ancient districts of Himachal like Kunindas (Kulindas), Oudumbars, Trigartas, Kulloots, Yodayas. Their time is from the second century BC to the second/third century AD. In Himachal, the Shavia-tradition of music can be directly performed in folk hymns sung in various local sub-languages. Traditional folklore is used in these folk hymns. The ascending-descending orders of musical notes used in these folk hymns are very simple and the shadow of many ragas can be seen.

Keywords

Himachal Pradesh, Shaiv Paranpra, Sangeet

\section{सार संक्षेपिका}

हिमाचल प्रदेश अपनी अनुठी संस्कृति व परम्पराओं के लिए जाना जाता है। हिमाचल के सभी जिलों में शिव की पूजा होती है। भगवान शिव को किसी न किसी रूप में पूजा जाता है। इतिहासकारों ने शैवधर्म की पुष्टि हिमाचल के प्राचीन जनपदों कुनिन्दों (कुलिन्द), औदुम्बरों, त्रिगर्तों, कुल्लूतों, यौधेयों द्वारा चलाए गए सिक्कों से की हैं। इनका समय ईसा पूर्व की दूसरी शताब्दी से ईसा पश्चात् की दूसरी तीसरी शताब्दी तक है। हिमाचल में शैव-परम्परा में संगीत के प्रत्यक्ष दर्शन यहां के विभिन्न स्थानीय उप-भाषाओं में गाए जाने वाले लोक-भजनों में किये जा सकते हैं। इन लोक भजनों में पारम्परिक लोकवाद्यों का प्रयोग किया जाता हैं। इन लोक भजनों में प्रयुक्त स्वरों का आरोही-अवरोही क्रम अत्यन्त सरल तथा अनेक रागों की छाया देखी जा सकती हैं।

बीज शब्द

हिमाचल प्रदेश, शैव-परम्परा, संगीत

\section{भूमिका}

भगवान शिव की कल्पना भारतीय संस्कृति की अद्भुत देन है। हिन्दू धर्म में शिव की उपासना अत्यन्त प्राचीन काल से प्रचलित है। शिव के उपासक शैव तथा उनसे सम्बन्धित धर्म को 'शैवधर्म' कहा जाता है। पुरातात्विक शोधानुसार सिन्धु सभ्यता जिस देवता की पूजा करते थे, वह वर्तमान शिव की है। मोहनजोदड़ो तथा हड़प्पा की खुदाइयों में प्राप्त वृषभ प्रतिमा को नन्दी का रूप माना जाता है। इस सभ्यता के अवशेषों में पशुओं से घिरे हुए ध्यानखय देवता की मूर्ति भी मिली है। सर जॉन मार्शल के कथनानुसार यह पशुपति शिव ही है। यह अनुमान है कि शिव आरम्भ में पशुओं का देवता रहा होगा, परन्तु बाद में आत्माओं का देवता बन गया होगा। आज भी हिमाचल प्रवेश में ऐसे देवता है जिन्हें गाय-बैल, भेड़-बकरी आदि पशुओं का देवता माना जाता है। आज भी कई जगहों पर अपनी मन्नत पूरी होने पर जंगली जानवरों के सींग मन्दिरों के मुख्य द्वारों पर लगाते हैं। हिमाचल प्रदेश में लोक धर्म की रूपरेखा आज भी वही है जो सिन्धु-सभ्यता के समय में थी। 


\section{हिमाचल प्रदेश में शैवधर्म}

\section{ऐतिहासिक पक्ष}

हिमाचल प्रदेश में अनेक प्रागैतिहासिक तथा पौराणिक जातियां निवास करती है। लोक देवताओं का प्रभुत्व तथा पूजा इस क्षेत्र की विशिष्ट संस्कृति है। जगमोहन बलोखरा अपनी पुस्तक "अलौकिक हिमाचल प्रदेश" में लिखते हैं कि हिमाचल प्रदेश में सिन्धु सभ्यता की समकालीन जातियां कोल, आदिम आग्नेय या निषाद वशी, किन्नर, नाग और खश थी। परन्तु यहां पर सबसे पहले जाति कौन थी यह निश्चित रूप से नहीं कहा जा सकता। कोल जाति यहां के मूल निवासियों में से एक कहे जाते हैं। इनका मुख्य व्यवसाय कृषि एवं पशुपालन था। हिन्दू धर्म में पशु देवता इसी प्राचीन जाति की देन है। कोल के बाद किरात जाति को ऋषि वशिष्ठ ने 'शिशनदेव' अर्थात् 'लिंग को देवता मानकर पूजने वाले' कहा है। ये जातियां हिमाचल की तराइयों में यत्र-तत्र बसती थी। यजुर्वेद तथा अथर्ववेद में इनका उल्लेख पर्वतीय गुफाओं के निवासियों के रूप में आया हैं। नाग जाति से सम्बन्धित कई गढ़ हिमाचल में मिलते हैं। ये मणिधारी सर्पों की पूजा करते थे। आज भी हिमाचल प्रदेश के नाग मन्दिरों में नाग का शासक रूप दिखाया गया है। एक अन्य जाति खश का पता यहां की परम्पराओं, लोक-गाथाओं, किंवदन्तियों, लोक-कथाओं तथा लोक-नाट्यों से चलता है। खश जाति से निकले कुलिन्दों ने अपने राज्य की स्थापना की।

इन जातियों के साथ शिव का घनिष्ठ सम्बन्ध रहा है इस बात की पुष्टि हमें धार्मिक जीवन की परम्पराओं, गुफाओं के अन्दर बने लिंगों एवं मूर्तियों तथा पुराने मन्दिरों में बनी प्रतिमाओं से होती है। डॉ० भण्डारकर का मानना है कि रूद्र-शिव का सम्बन्ध आरम्भ में जंगली जातियों से रहा होगा। शिव का भांग धतूरा पीना, गले में सांप और मृगछाला आदि बातें, वनवासियों के ही संस्कार से आई होगीं, जो नाग पूजक रहें होगें। हिमाचल प्रदेश के इतिहास के स्रोतों में सिक्कें आर्थिक, राजनीतिक, धार्मिक स्थिति का चित्रण प्रस्तुत करते हैं। यहां पर उपलब्ध हुई मुद्रायें त्रिगर्तों, औदुम्बरों, कुलिन्दों, कुल्लूतों आदि प्राचीन जनपदों से सम्बन्धित हैं। इनका समय ईसा पूर्व की दूसरी शताब्दी से ईसा पश्चात् की दूसरी-तीसरी शताब्दी तक है।

कुनिन्द

कुनिन्दों का ज्ञान हमें साहित्य एवं पुरातत्व दोनों स्रोतों से मिलता हैं। महाभारत, मार्कण्डेय पुराण, वायु पुराण, विष्णु पुराण में उन्हें कुलिन्द कहा गया हैं। विष्णु पुराण में उन्हें ककुलिंदोपत्यका' अर्थात् पर्वतीय घाटियों में रहने वाले (कुनिन्द) कहा गया है। कुनिन्द पहाड़ी लोग थे जो व्यास, सतलुज और यमुना नदी के बीच क्षेत्र (शिमला, सिरमौर) तथा अम्बाला, सहारनपुर के बीच रहते थे। इस जनपद से सम्बन्धित रोचक तथ्य उनके सिक्कों से मिलते हैं। ये सिक्कें हमीरपुर, ज्वालामुखी, सहारनपुर, अम्बाला और गढ़वाल तक के क्षेत्र में मिले है। इन सिक्कों को दो श्रेणियों में बांटा गया है। प्रथम श्रेणी के सिक्कों में ब्राह्मी लिपि और प्राकृत भाषा में राज्ञा: कुनिन्दस्य अमोधभूतिएय 
महाराजस्य' तथा अन्य सिक्कों पर 'अमोधभूतस महाराजस्य राजकुणदस' अंकित है। दूसरी तरफ लक्ष्मी की मूर्ति, वृक्ष, मृग कुछ अन्य चिन्ह अंकित हैं।

दूसरी श्रेणी के सिक्कों में छत्रोश्वर के सिक्कें कुषाण शैली के हैं। इन सिक्कें पर ब्राह्मी लिपि में 'भगवतो छत्रोश्वर महात्मनह' तथा बीच में जटाजूट शिव की मूर्ति अंकित है। सिक्के के दूसरी तरफ मृग, नन्दी पद, सर्प, स्वस्तिक चिन्ह अंकित हैं।

अंतः स्पष्ट है कि कुनिन्दों का प्रधान देवता शिव था और वे उसे छत्रोश्वर कहते थे। जो छत्र की तरह उनके राज्य की रक्षा करता था। अमोद्यभूति की मुद्रायें ईसा पूर्व काल तथा छत्रोश्वर की मुद्रायें तीसरी शताब्दी की है।

औदुम्बर

औदुम्बर प्राचीन हिमाचल प्रदेश के सबसे प्रसिद्ध कबीले के लोग थे। मार्कण्डेय, चान्द्र व्याकरण, विनयपिट्फ, हरिवंश, सरस्वती कण्ठाभरण, वायु और ब्रह्माण्ड पुराण में इसका उल्लेख उपलब्ध होता है। इस जनपद से सम्बन्धित सर्वाधिक ज्ञान हमें कांगड़ा जिले के ज्वालामुखी, होशियारपुर और पठानकोट में मिले सिक्कों से प्राप्त होता है। इन सिक्कों को तीन श्रेणियों में बांटा जा सकता है। प्रथम श्रेणी में तांबे के चौकोर के सिक्कों पर शिव की आकृति, त्रिशूल, शिवालय, वृषभ चिन्ह अंकित हैं, जो शैवधर्म से सम्बन्धित है। इन्हें ईसा पूर्व प्रथम शती का माना गया है। दूसरी श्रेणी की चांदी की मुद्राओं पर शिवदास, रूद्रदास, घरघोषस और भागवत महादेव के नाम आते हैं। इन मुद्राओं पर खरोष्ठी एवं ब्राह्मी लिपि में 'महादेवस राणा शिवदासस औदुम्बरिस', 'महादेवस राणा रूद्रदासस औदुम्बरिस', 'महादेवस राणा घरघोषस औदुम्बरिस' तथा 'भागवत महादेवस राणा औदुम्बरिस' अंकित हैं। विद्वानों ने इन मुद्राओं को ईसवीं प्रथम शताब्दी का माना है। तीसरी श्रेणी की मुद्राएं आर्यमित्र, अजयमित्र, महीमित्र, भानुमित्र तथा महाभूतिमित्र शासकों द्वारा चलाई गई हैं। इनका समय प्रथम शताब्दी का उतरार्द्ध है। उपरोक्त मुद्राओं पर अंकित शिव की आकृति, त्रिशूल, वृषभ तथा राजाओं के नामों से सिद्ध होता है कि औदुम्बर शैवमतानुयायी थे।

उपरोक्त जनपदों के अतिरिक्त यौधेयों के सिक्कों से भी हिमाचल में शैवधर्म की पुष्टि होती है। इन सिक्कें पर खड़े शिव की आकृति मिलती है, एक हाथ में त्रिशूल, दूसरे में कुठार तथा कमर मृग चर्म पहने हुए है। इसके अतिरिक्त सिक्कों पर शैवधर्म के 'नन्दी वृषभ' प्रतीक भी मिलते हैं, शक-कुषाण भी शिव उपासक थे। कुषाण के सिक्कों पर शिव सूर्य, अग्नि तथा बुद्ध की आकृतियां हैं। इन शासकों की तरह कश्मीर का हूण राजा मिहिरकुल और थानेश्वर के सम्राट हर्षवर्धन के पूर्वज भी शैव थे। शैव धर्म के विकास की यह स्थिति गुप्त काल से पूर्व की है क्योंकि औदुम्बरों, कुनिन्दों तथा यौधेयों के राज्य का पतन हो चुका था। तृतीय शताब्दी ई0 पू० से लेकर चौथी शती ई0 तक शैवधर्म प्रदेश के विभिन्न भागों में फैल चुका था। प्रदेश के कुल्लू-भरमौर के वर्मन शासक, तथा स्पीति के सेनवंश भी शैव थे। 


\section{सांगीतिक पक्ष}

हिमाचल की धरती शिव की निवास भूमि और क्रीड़ा स्थली है। यहां के सभी जिलों में शिव की पूजा होती है। यहां के स्थानीय लोग अपनी स्थानीय भाषा में भगवान शिव को महादेव, महेश्वर, भूतनाथ, सदाशिव, कैलाश पति तथा लोक देवता के रूप में शिर्गुल, धुडु, महासु, देऊ इत्यादि नामों से पुकारते हैं। शिव-विवाह को स्थानीय भाषा में ईशर, छड़ाह, शिवीण इत्यादि कहते हैं। शैवधर्म की प्राचीन परम्परा आज भी जिला चम्बा के भरमौर क्षेत्र में देखी जा सकती है। यहां के मूल निवासी गद्दी शिव भक्त हैं। यहां भगवान शिव को प्रसन्न करने के लिए एक विशेष पूजन किया जाता है। जिसे स्थानीय भाषा में 'नवाला' कहा जाता है। इस पारम्परिक नवाला गायन में ढोलकी, कांस की थाली, घड़ा आदि लोकवाद्य तथा रातभर डंडारस नृत्य करते हैं। हिमाचल प्रदेश के अन्य जिलों में भी शैव-परम्परा की पारम्परिकता लोक भजनों में जीवंत है। शैव-परम्परा से सम्बंधित 'नवाला गायन' स्वरलिपि सहित प्रस्तुत किया जा रहा हैं

लोक भजन के बोल

$$
\begin{aligned}
& \text { "आ सामियाँ आ बै वो सामियाँ, } \\
& \text { ले बो सामि अपणे ओधारो } \\
& \text { भोलेया सामियाँ। } \\
& \text { अस्सी वो कोठे तेरा मण्डल सजे } \\
& \text { ले बो सामि अपणे ओधारों } \\
& \text { भोलेया सामियाँ। } \\
& \text { चोले ओ माऐं तेरा मण्डल सजे } \\
& \text { ले बो सामि अपणे ओधारों } \\
& \text { भोलेया सामियाँ। } \\
& \text { अक अरज मेरी होर होणे } \\
& \text { भुखयाँ जो भोजन दीऐं } \\
& \text { भोलया सामियाँ। } \\
& \text { अक अरज मेरी होर होणे } \\
& \text { अन्धयां जो लोचण लाऐं } \\
& \text { भोलेया बो सामियाँ।" }
\end{aligned}
$$

भावार्थः यह शैव-परम्परा का अति प्राचीन भजन है। यह गद्दी समुदाय द्वारा नवाला उत्सव में भगवान शिव के आवाहन के लिए गाया जाता है। इन पंक्तियों में शिवजी के प्रति आभार व्यक्त कर उन्हें स्थान ग्रहण करने की प्रार्थना की जाती हैं। अगली पंक्तियों में भूखों को अन्न तथा अन्धों को रोशनी प्रदान करने की प्रार्थना की है। 


\section{स्वरलिपि}

स्थाई

\begin{tabular}{|c|c|c|c|c|c|c|c|c|c|c|c|c|}
\hline 1 & 2 & 3 & 4 & 5 & 6 & 7 & 8 & 9 & 10 & 11 & 12 & 13 \\
\hline ग & ग & ग & ग & गरे & प & प & ग & ग & रे & रे & ग & रे \\
\hline आ & $S$ & सा & मि & याऽ & आ & $S$ & बै & वो & सा & मि & याँ & $S$ \\
\hline सारेग & सा & सा & सा & प̣ & सा & $S$ & साग & ग & रेरे & सरे & गप & गरे \\
\hline लेबोऽ & सा & मि & $\begin{array}{l}\text { अ } \\
2\end{array}$ & प & णे & $S$ & $\begin{array}{l}\text { ओधा } \\
0\end{array}$ & $S$ & रोऽ & $\begin{array}{l}\text { भोले } \\
3\end{array}$ & याS & सामि \\
\hline
\end{tabular}

अन्तरे भी इन्हीं स्वरों के आधार पर गाए जाएगें।

यह लोक भजन राग पहाड़ी में स्वरबद्ध हैं। यह बिलावल थाट से जनित राग है। इसकी जाति औढ़व-औढ़व है तथा दीपचन्दी ताल में तालबद्ध हैं।

\section{निष्कर्ष}

हिमाचल प्रदेश में शैवधर्म का विकास गुप्तोत्तर काल में भी हुआ। इस काल में सम्पूर्ण प्रदेश इस धर्म से सम्बन्धित कला से भर गया। कांगड़ा, कुल्लू और लाहौल ये तीनों गुप्त काल में शैवधर्म के प्रधान केन्द्र थे। यहां के अधिकतर प्राचीन मन्दिर इसी काल में बनाये गये, जिन्हें शैव प्रतिमाओं तथा अन्य हिन्दू देवी-देवताओं से अलंकृत किया गया। हिमाचल में विविध नामों से पूजे जाने वाले शिव किसी-न-किसी रूप में जन-जन के मन में अपना स्थान बनाए हुए हैं। पौराणिक कथाओं के आधार पर यहां के लोगों की देवी-देवताओं में आस्था और भी गूढ़ हो गई। यहां शिव के प्राचीनतम स्मृति अवशेष इसकी चोटियों किन्नर कैलाश, श्रीखण्ड, मणिमहेश, चूड़धार इत्यादि पर हैं। आज भी धर्म-विश्वास की दृष्टि से भरमौर के निवासी गद्दी शिव उपासक है। इस क्षेत्र में आज भी प्रत्येक घर में शिव पूजा की जाती है। जिसे गद्दी अपनी भाषा में 'नवाला' कहते हैं। हिमाचल प्रदेश के गांव-गांव और घाटी-घाटी में शिव -शक्ति के अनेक मन्दिर हैं। जिससे यह स्पष्ट होता है कि हिमाचल का प्राचीनतम धर्म शैवधर्म था और आज भी शैवधर्म हैं।

\section{सन्दर्भ ग्रन्थ}

बलोखरा, जगमोहन (2015). अलौकिक हिमाचल प्रदेश, एच. जी. पब्लिकेशन, दिल्ली। कश्यप, डॉ. पदम चन्द्र (1986). हिमाचली संस्कृति का इतिहास, आर्य प्रकाशन मण्डल, दिल्ली। शर्मा डॉ. बंशी राम, (1986). हिमाचल लोक संस्कृति के स्रोत, आर्य प्रकाशन मण्डल, दिल्ली। सिहं मियां गोवर्धन (1996). हिमाचल प्रदेश का इतिहास, रिलायेन्स पब्लिशिंग हाऊस, नई दिल्ली। पांडे, डॉ. एल.पी. (1981). एनशिएंट हिमाचल हिस्टी, रिलीजन एंड कल्चर. श्रीमती कामिनी गुप्त, पंचशील पार्क, नई दिल्ली।

\section{साक्षात्कार}

शर्मा, पं. संसार, गांव व डाकघर उतराला, तहसील बैजनाथ, जिला कांगड़ा (हि० प्र०) 